\title{
Design of an undervoltage load shedding scheme for the Hydro-Québec system
}

\author{
Daniel Lefebvre \\ Hydro-Québec, Division TransEnergie \\ Complexes Desjardins, Tour de 1’Est \\ CP 10000 Montreal (QC), Canada \\ lefebvre.daniel.4@hydro.qc.ca
}

\author{
Cédric Moors \\ Thierry Van Cutsem \\ Research Director, FNRS \\ University of Liège \\ Dept. of Elec. Eng. and Comp. Sc. \\ Sart Tilman B37 B-4000 Liège, Belgium \\ c.moors@ulg.ac.be
}

\begin{abstract}
This paper deals with the control logic of an undervoltage, closed-loop load shedding scheme aimed at protecting the Hydro-Québec system against long-term voltage instability. This scheme relies on a set of "if-then" rules whose parameters are determined through combinatorial optimisation, relying on the system dynamic response over a set of scenarios. Preliminary results of the above optimisation technique are given, besides a brief description of the foreseen implementation.
\end{abstract}

\section{INTRODUCTION}

There are two lines of defence against incidents likely to trigger system instability:

- preventively: estimate security margins with respect to credible contingencies, i.e. incidents with a relatively high probability of occurrence. Very often, preventive security criteria state that the system should respond in an acceptable way to (N-1)-type incidents, without the help of post-contingency actions affecting generators and/or loads;

- correctively: implement System Protection Schemes (SPS) (also referred to as Special Protection Schemes), to face the more severe, but less likely incidents. The latter are typically $\mathrm{N}-2$ or more dangerous disturbances.

This paper focuses on corrective control of long-term voltage instability, driven by load tap changers, generator overexcitation limiters, switched shunt compensation, restorative loads, and possibly secondary voltage control [1], [2]. This type of instability has become a major threat in many systems.

While it should be used in the last resort, load shedding is an effective countermeasure against voltage instability [3], especially when the system undergoes a severe initial voltage drop that cannot be tolerated for a long time.

As for any SPS, the design of a load shedding scheme is a challenging task in terms of number of possible protection settings and (pre- and post-disturbance) scenarios to consider. Even study engineers with a very good knowlegde of their system face this huge complexity problem. Tools are thus needed to help them choosing the most appropriate designs.

This paper reports on the use of combinatorial optimisation for the tuning of a closed-loop load shedding controller aimed at protecting the Hydro-Québec system against long-term voltage instability. It is the continuation of the previous publications [4], [5], [6], considering a new controller structure, new criteria and new dimensioning scenarios.

\section{UNDERVOLTAGE LOAD SHEDDING IN THE HYDRO-QUÉBEC SYSTEM}

With its long transmission corridors between the hydro generation areas in the North and the main load centers in the South part of the province (see Fig. 1), the Hydro-Québec (H-Q) system is exposed to angle, frequency and voltage stability problems.

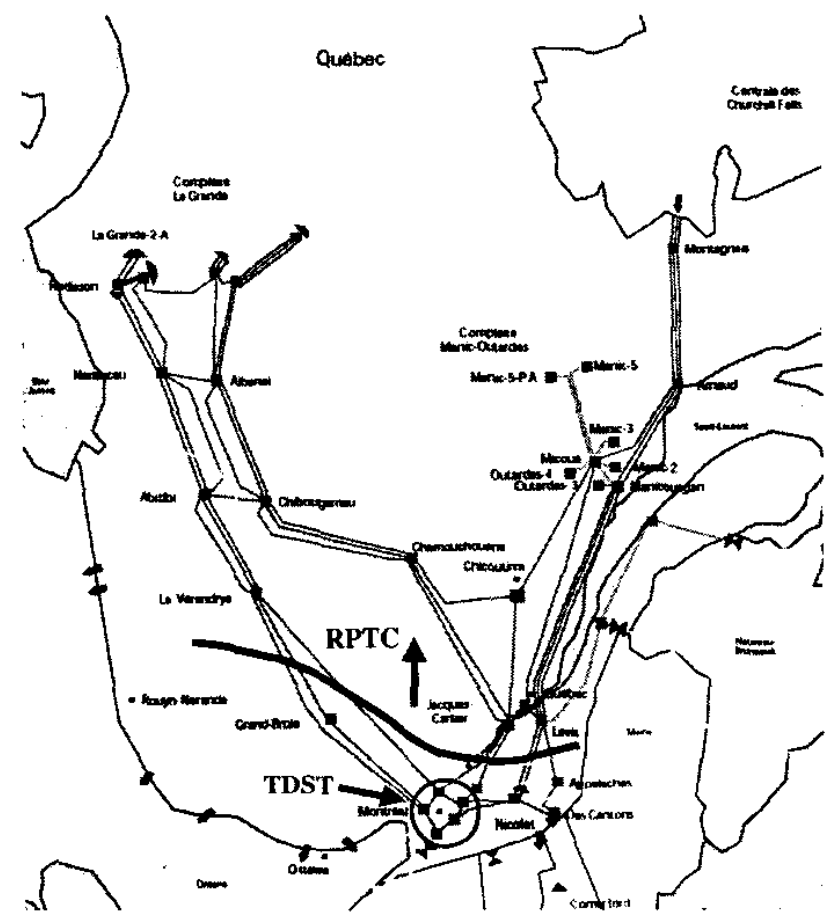

Fig. 1. Hydro-Québec EHV system with RPTC and TDST action zones

Besides static var compensators and synchronous condensers, the automatic shunt reactor switching devices - named 
MAIS - play an important role in voltage control [7]. These devices, in operation since early 1997, are now available in twenty-two $735-\mathrm{kV}$ substations and control a large part of the total 25,500 Mvar shunt compensation. Each MAIS device relies on the local voltage, the coordination between substations being performed through the switching delays. While fast-acting MAIS can improve transient angle stability, slower MAIS significantly contribute to voltage stability. MAIS devices react to voltage drops but also prevent overvoltages by reconnecting shunt reactors when needed.

In order to upgrade the reliability of its transmission system, $\mathrm{H}-\mathrm{Q}$ has developed over the recent years an extensive defence plan against major disturbances. Besides traditional underfrequency load shedding measures, an extensive generation rejection and remote load shedding scheme - named RPTC - has been installed to face transient angle stability problems. The next step of this deployment is the undervoltage load shedding scheme - named TDST - whose implementation is scheduled for the beginning of 2004 (see Fig. 1).

While RPTC is an event-based SPS (due to the speed of angle instability phenomena), TDST will be response-based [8] (owing to the nature of long-term voltage instability), relying on transmission voltages measured in the Montreal area. More precisey, local voltages will be measured in five $735-\mathrm{kV}$ substations equipped with MAIS devices and validated through the data acquisition chains of the latter. The measurement sampling rate will be 0.1 second. The average $\bar{V}$ of these local voltages will then be considered, provided that three valid values out of the five have been received.

The protection will rely on $\bar{V}$ not only to allow bad data rejection but also to better identify dangerous disturbances. Indeed, while an N-1 contingency (for which no load shedding is allowed) can affect one of the local voltages, it will have little effect on the average $\bar{V}$. Conversely, a significant drop of $\bar{V}$ is an indication that an $\mathrm{N}-2$ or more severe disturbance has occured.

The shedding logic will rely on the absolute value of $\bar{V}$, as well as on the sudden decreases $\Delta \bar{V}$ of this signal. In normal operating conditions, the voltage profile of the $\mathrm{H}-\mathrm{Q}$ system is maintaind in a narrow range around its nominal value (typically [0.98 1.02] pu) while the highest shedding threshold will be around $0.94 \mathrm{pu}$ (a typical value of the critical voltage in this capacitive system). The $\Delta \bar{V}$ criterion is used to account for cases where pre-disturbance voltages would be at the upper limit of the above range. The voltage drop $\Delta \bar{V}$ is computed with respect to a reference $V^{o}$ obtained by taking the average value of $\bar{V}$ over a sliding time window (of typically $30 \mathrm{~s}$ ). As soon as $\bar{V}$ will drop by more $1 \%$, the average value of $\bar{V}$ over the last available window will be taken as the reference $V^{\circ}$. Invalid values of $\vec{V}$ are discarded when computing $V^{\circ}$.

TDST will act in a pre-defined "load basin", of which it will be allowed to shed at most a certain percentage. The set of distribution circuit breakers that can be opened is known by the remote load shedding controller also used by RPTC.

By relying on $\bar{V}$, the protection is aimed at counteracting system-wide instability. In addition to this, local load shedding controllers will be attached to each of the five substations from which local voltage measurements are taken. Each of these controllers can act on a part of the above mentioned bassin. Their action will, however, be conditioned to some decrease of $\bar{V}$. These local controllers are not considered in the remaining of this paper.

\section{THE UNDERVOLTAGE LOAD SHEDDING LOGIC}

The control logic relies on the following:

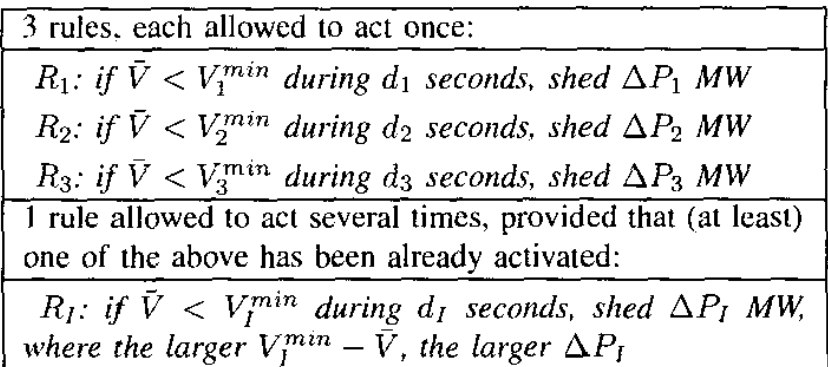

Rules $R_{1}$ to $R_{3}$ aim at making the voltage promptly recover once it has dropped below an unacceptable level (e.g. from the customer viewpoint). The $V_{1}^{\text {min }}$ parameter is set at this level, while the other voltage thresholds are such that :

$$
V_{3}^{\text {min }}<V_{2}^{\text {min }}<V_{1}^{\text {min }}
$$

Each rule corresponds to a different level of severity. Correspondingly, the load shedding amounts and delays are chosen so that the deeper the voltage drop, the larger the shedding:

$$
\Delta P_{3}>\Delta P_{2}>\Delta P_{1}
$$

and the shorter the delay:

$$
d_{3}<d_{2}<d_{1}
$$

Alternative choices may be thought of. For instance, there might be less or more rules, although in the latter case, the computational effort of the optimisation should be kept in mind. Also, the inequalities $(2,3)$ are not mandatory, although they have been found to work well.

The idea behind rule $R_{I}$ is to set up a controller adjusting its action to the severity of the situation. The latter is assessed through the average voltage drop over the time interval $d_{I}$. To this purpose, the load shedding step is given by:

$$
\Delta P_{I}=k \Delta \vec{V}_{a v g} \quad \text { with } \quad \Delta P_{I}^{\min } \leq \Delta P_{I} \leq \Delta P_{I}^{\max }
$$

where $\Delta \bar{V}_{a v g}$ is the average voltage drop :

$$
\Delta \bar{V}_{\mathrm{avg}}=\frac{1}{d_{I}} \int_{t_{0}}^{t_{0}+d_{I}}\left(V_{I}^{\text {min }}-\bar{V}\right) d t
$$

and $t_{o}$ is the time at which $\bar{V}$ falls below $V_{I}^{\text {min }}$. Clearly, the larger $V_{I}^{\text {min }}-\bar{V}$, the larger $\Delta P_{I}$.

Finally, $\Delta P_{I}$ is discretized as indicated in Fig. 2, to take into account that loads are shed by blocks (when opening circuit breakers).

While the main purpose of $R_{1}, R_{2}, R_{3}$ is to react to a severe voltage drop, the role of $R_{I}$ is the final stabilization of the 


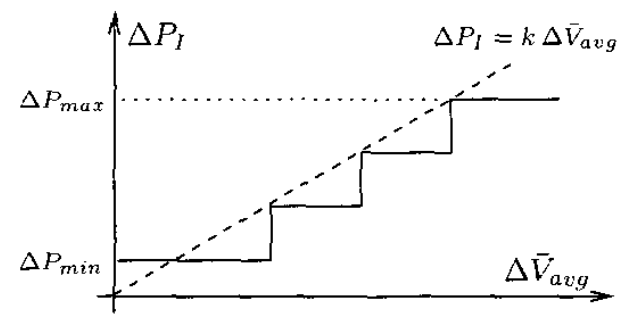

Fig. 2. Discretization of load shedding

system. In this respect, the thresholds of $R_{1}$ and $R_{I}$ are such that:

$$
V_{1}^{\min }<V_{I}^{\min }
$$

With $V_{I}^{\text {min }}$ set to a higher value, the risk increases of an undue load shedding following a large system transient. On the basis of simulations, it is possible to tune the protection parameters to avoid such false alarms; however, the uncertainty affecting the simulation models must be considered as well. Therefore, to increase the protection security, H-Q has decided to condition the application of $R_{I}$ to the previous triggering of (at least) one of the rules $R_{1}$ to $R_{3}$.

Rules $R_{1}, R_{2}, R_{3}$ are "concurrent" in the sense that any of them can be applied irrespective of the others. However, each rule may be triggered only once.

On the other hand, $R_{I}$ is conditioned to the other rules, as explained above, but can be applied repetitively. This yields a closed-loop design since the protection may act several times, each action being based on the measured result of the previously taken actions, and adjusted in amplitude to the system evolution. This closed-loop design guarantees a higher SPS robustness against modelling uncertainties at the design stage.

Note finally that by adjusting its action to the severity of the situation, the controller minimizes the risk of overfrequency (and thermal unit tripping) due to excessive load shedding.

\section{OPTIMISATION OF THE LOAD SHEDDING CONTROLLER}

The methodology used to adjust the settings of TDST consists of two steps [6]. In the first step, a set of training scenarios is built, and each unstable scenario of this set is analyzed to determine the minimal load shedding needed. In the second step, the protection parameters are adjusted in order to approach as closely as possible the optimal sheddings computed in the first step, over the whole set of scenarios. A combinatorial optimization method is used to this purpose.

\section{A. Scenario analysis}

The first step thus consists in setting up a set of $s$ training scenarios, corresponding to various topologies, load levels, generation schemes, contingencies, etc. Given the load basin that TDST will control, for each scenario, we determine $P_{i}^{\star} \quad(i=1, \ldots, s)$, the minimal amount of load to shed at a single point in time.
To this purpose, for a given shedding delay $\tau$, the minimal amount $P_{i}^{\text {min }}$ of load to shed is determined iteratively by incremental or binary search [6]. This determination is repeated for various values of $\tau ; P_{i}^{\star}$ is then taken as the minimum of the $P_{i}^{\text {min }}(\tau)$ curve.

An example of such a curve is given in Fig. 3 for the HydroQuébec system. For the scenario of concern, the best time to shed load is 15 to 20 seconds (or 35 to 40 ) seconds after the disturbance. This delay allows the MAIS to trip shunt reactors and hence to increase the network transmission capability, thereby reducing the amount of load to shed. Shedding earlier resets the MAIS by increasing the transmission voltages monitored by these devices. The value of $P_{i}^{\star}$ is $650 \mathrm{MW}$.

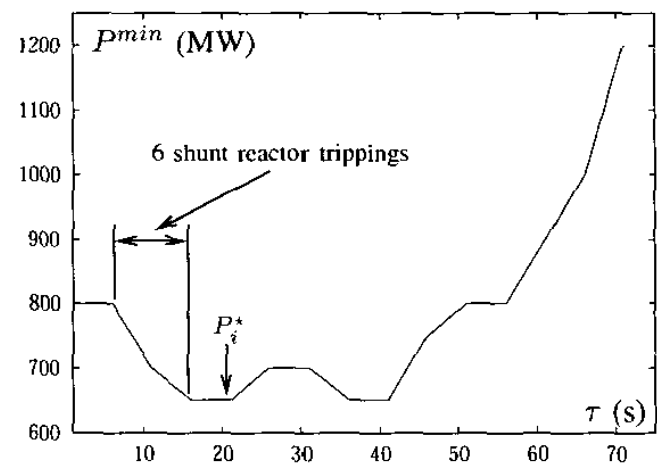

Fig. 3. minimal load shedding vs. shedding delay in a given scenario

\section{B. Statement of the optimization problem}

Let us denote by $\mathbf{x}$ the vector of all parameters which appear in the rules and hence have to be adjusted:

$$
\begin{aligned}
\mathbf{x}= & {\left[\Delta P_{1} \Delta P_{2} \Delta P_{3} d_{1} d_{2} d_{3} V_{2}^{\text {min }} V_{3}^{\text {min }}\right.} \\
& \left.k \Delta P_{I}^{\text {min }} \Delta P_{I}^{\text {max }} V_{I}^{\text {min }} d_{I}\right]
\end{aligned}
$$

Given the $s$ training scenarios, the problem is to determine $\mathbf{x}$ such that the following requirements are met:

1) the amount of load shedding must be as close as possible to the ideal value $P_{i}^{\star}$ determined in the first step;

2) all unstable scenarios must be saved (dependability);

3) no load must be shed in a stable scenario (security);

4) the average voltage $\bar{V}$ must not stay below $V_{1}^{\text {min }}$ for more than some time.

This can be translated into an optimization problem: minimize either the $L_{1}$ objective:

$$
\frac{1}{s} \sum_{i=1}^{s}\left[P_{i}^{s h}(\mathbf{x})-P_{i}^{\star}+p_{i}(\mathbf{x})\right]
$$

or the $L_{\infty}$ objective:

$$
\max _{i}\left[P_{i}^{s h}(\mathbf{x})-P_{i}^{\star}+p_{i}(\mathbf{x})\right]
$$

where, in the $i$-th scenario, $P_{i}^{s h}(\mathbf{x})$ is the total load power shed by the controller and $p_{i}(\mathbf{x})$ is a penalty term accounting 
for the violation of the above requirements. In Eq. (8) (resp. (9)), the sum (resp. the max) extends over the unstable scenarios. The expression within brackets is expected to be positive since:

- $P_{i}^{s h}(\mathbf{x})>P_{i}^{\star}$ : indeed, more load has to be dropped when shedding in several steps (as the controller docs) rather than a single one (as assumed when computing $P_{i}^{*}$ ). This assertion has been verified in all our simulations;

- $p_{i}(\mathbf{x}) \geq 0$. For details about the choice of the penalties, please refer to [5], [6].

\section{The Branch-and-Bound approach}

The above optimization problem is complex. Indeed, both $P_{i}^{s h}$ and $p_{i}$ must be determined from time-domain simulations and hence, explicit analytical expressions cannot be established. Moreover, they vary with $\mathbf{x}$ in a discontinuous manner, which prevents from using mathematical programming methods. Finally, multiple local minima are expected. This is why we prefer to resort to combinatorial optimization.

To this purpose, each component of $\mathbf{x}$ is discretized in a finite number of possible values. The discretization steps are chosen in accordance with the engineering knowledge of the problem (see Section V.A).

Evaluating the $L_{1}$ or $L_{\infty}$ objectives for a given protection setting requires to simulate the $s$ scenarios (in order to compute the $s$ terms $\left.P_{i}^{s h}(\mathbf{x})-P_{i}^{\star}+p_{i}(\mathbf{x})\right)$. This time-consuming step precludes a brute-force enumeration of all the discrete instances of $\mathbf{x}$.

Luckily, a short-cut can dramatically decrease the computational effort. It consists, during the enumeration of the various instances of $\mathbf{x}$, in keeping track of $I_{b}$, the best value of the objective obtained so far. $I_{b}$ is an upper bound on the sought global minimum. Now, as the various scenarios are being checked for a given instance of $\mathbf{x}$, the objective function can only increase. Therefore, as soon as the objective function becomes greater than $I_{b}$, the scenario enumeration can be broken and the current instance of $\mathbf{x}$ abandoned; otherwise, the value of the objective becomes the new $I_{b}$. This procedure is outlined in Fig. 4 , where $\mathbf{x}^{(j)}$ denotes the $j$-th instance of $\mathbf{x}(j=1, \ldots, N)$.

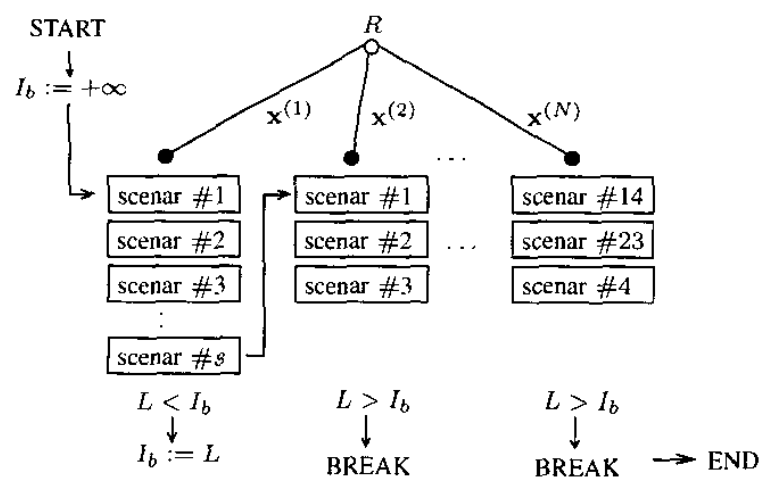

Fig. 4. sketch of the Branch-and-Bound method
This significant short-cut of the enumerative search is nothing but an application of the Branch-and-Bound principle [9]. Note that when the load shedding controller only involves rules of the type $R_{1}, R_{2}, R_{3}$, the optimization problem can be formulated as a tree exploration and an improved bound can be built, from which further speed-up can be obtained with the Branch-and-Bound method [6].

When using the $L_{1}$ objective, the gain in computing time is expected to be smaller. Indeed, due to the additive nature of this objective, a higher number of scenarios have usually to be simulated before the objective function reaches the $I_{b}$ value. This is a drawback of the $L_{1}$ objective. On the other hand, this objective usually behaves more smoothly, i.e. is less sensitive to small changes in parameters [6].

It is easily seen from the above description that the performance will be improved if $I_{b}$ decreases at an early stage of the search and/or the scenarios with the largest contribution to the objective are processed first. As regards the second aspect, it may be advantageous to dynamically reorder the scenarios on the basis of their ability to break enumerations, observed at the beginning of the search or in previous optimizations [6].

\section{Distributed processing}

In spite of the effectiveness of the Branch-and-Bound algorithm, the computational burden may remain prohibitive, especially when the $L_{1}$ objective is considered or when the size of the search space to explore is important. Fortunately, the very structure of the problem makes it easy to distribute computations on several "slave" processors coordinated by a "master" one.

In the first scheme to come to mind the master assigns the simulation of a scenario to each slave (as soon as it becomes available) and receives from the latter the value of $P_{i}^{\text {sh }}$ and the stable/unstable diagnosis. Enumeration breaks and $I_{b}$ updates are taken care of by the master. This scheme is efficient in so far as the time for transferring the data from the master to a slave is small compared to the simulation time, so that the communication overhead remains negligible.

An alternative consists of using each slave to explore a subset of instances of $\mathbf{x}$. In this second scheme, as soon as $I_{b}$ decreases, the new value must be broadcasted to all the other slaves.

\section{E. Time simulation tools}

Detailed long-term voltage instability simulations remain time consuming. This computational burden does not exist with the Quasi Steady-State (QSS) simulation, a welldocumented simplified long-term simulation technique [2].

QSS simulation is useful for processing a large search space and taking preliminary decisions, for instance deciding which parameters will be subsequently fixed, to save computing time.

An example of comparison between QSS and detailed simulations is given in Fig. 5 , in an unstable case without load shedding. As can be seen, the long-term behaviour is well captured by the QSS simulation but, expectedly, the shortterm transients (taking place over the first 20 seconds) are not 
reproduced. This period of time where voltage and frequency experience large transients has to be simulated in detail in order to properly set the protection parameters.

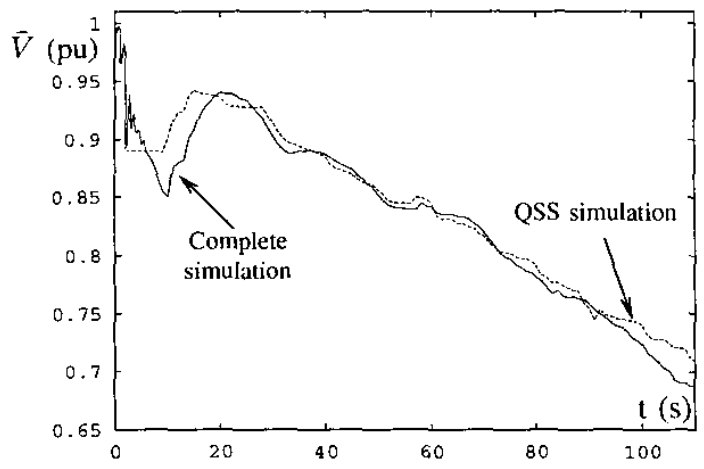

Fig. 5. QSS vs. detailed simulation (unstable case)

In this respect, the coupling between full and QSS simulations presented in [10] is a very interesting compromize between efficiency and accuracy. The idea is to simulate the short-term period using detailed simulation and, once the transients have died out, to switch to QSS simulation, the latter being initialized "out of equilibrium".

\section{RESULtS}

\section{A. Training scenarios and criteria}

The study reported in this paper involves 22 system configurations, summarized in Table I.

TABLE I

SYSTEM CONFIGIURATIONS CONSIDERED IN THE TRAINING SCENARIOS

\begin{tabular}{|c|c|c|c|c|}
\hline $\begin{array}{c}\text { confi- } \\
\text { guration }\end{array}$ & $\begin{array}{c}\text { number of } \\
\text { out of service }\end{array}$ & $\begin{array}{c}\text { synchronous } \\
\text { condensers }\end{array}$ & $\begin{array}{c}\text { MAIS } \\
\text { devices }\end{array}$ & $\begin{array}{c}\text { load active } \\
\text { power } \\
\text { exponent }\end{array}$ \\
\hline A & 0 & 6 & 6 & 1 \\
B & 1 & 6 & 6 & 1 \\
C & 1 & 8 & 6 & 1 \\
D & 1 & 8 & 6 & 1 \\
E & 1 & 7 & 4 & 1 \\
F & 1 & 8 & 10 & 1 \\
G & 1 & 7 & 6 & 1 \\
H & 4 & 8 & 6 & 1 \\
I & 4 & 7 & 4 & 1 \\
J & 3 & 8 & 10 & 1 \\
K & 3 & 7 & 6 & 1 \\
L & 3 & 8 & 10 & 1 \\
M & 3 & 7 & 6 & 1 \\
N & 1 & 7 & 3 & 1 \\
O & 1 & 7 & 4 & 1 \\
P & 1 & 7 & 4 & 1 \\
Q & 3 & 7 & 5 & 1 \\
R & 0 & 6 & 6 & 1.3 \\
S & 1 & 8 & 6 & 1.3 \\
T & 1 & 8 & 6 & 1.3 \\
U & 4 & 8 & 6 & 1.3 \\
V & 3 & 7 & 6 & 1.3 \\
\hline
\end{tabular}

Table II details the 38 scenarios finally selected. They involve $\mathrm{N}-1, \mathrm{~N}-2$ and $\mathrm{N}-3$ contingencies, respectively. In accordance with the standard operating rules, the system is stable following any $\mathrm{N}-1$ incident. The MAIS devices can be used to this purpose.

TABLE II

DESCRIPTION OF THE 38 TRAINING SCENARIOS

\begin{tabular}{|c|c|c|c||c|c|c|c|}
\hline No & conf. & $\begin{array}{c}\text { incid. } \\
\text { type }\end{array}$ & $\begin{array}{c}P_{i}^{*} \\
(\mathrm{MW})\end{array}$ & No & conf. & $\begin{array}{c}\text { incid. } \\
\text { type }\end{array}$ & $\begin{array}{c}P_{i}^{*} \\
(\mathrm{MW})\end{array}$ \\
\hline 1 & $\mathrm{~A}$ & $\mathrm{~N}-1$ & 0 & 20 & $\mathrm{G}$ & $\mathrm{N}-2$ & 300 \\
2 & $\mathrm{~A}$ & $\mathrm{~N}-1$ & 0 & 21 & $\mathrm{O}$ & $\mathrm{N}-2$ & 100 \\
3 & $\mathrm{R}$ & $\mathrm{N}-2$ & 650 & 22 & $\mathrm{P}$ & $\mathrm{N}-2$ & 300 \\
4 & $\mathrm{~A}$ & $\mathrm{~N}-2$ & 400 & 23 & $\mathrm{H}$ & $\mathrm{N}-1$ & 0 \\
5 & $\mathrm{R}$ & $\mathrm{N}-3$ & 1100 & 24 & $\mathrm{H}$ & $\mathrm{N}-1$ & 0 \\
6 & $\mathrm{~B}$ & $\mathrm{~N}-1$ & 0 & 25 & $\mathbf{l}$ & $\mathrm{N}-2$ & 300 \\
7 & $\mathrm{~B}$ & $\mathrm{~N}-2$ & 400 & 26 & $\mathrm{U}$ & $\mathrm{N}-2$ & 1450 \\
8 & $\mathrm{C}$ & $\mathrm{N}-1$ & 0 & 27 & $\mathrm{H}$ & $\mathrm{N}-2$ & 1200 \\
9 & $\mathrm{C}$ & $\mathrm{N}-2$ & 200 & 28 & $\mathrm{U}$ & $\mathrm{N}-2$ & 650 \\
10 & $\mathrm{~S}$ & $\mathrm{~N}-2$ & 1250 & 29 & $\mathrm{~J}$ & $\mathrm{~N}-1$ & 0 \\
11 & $\mathrm{C}$ & $\mathrm{N}-2$ & 550 & 30 & $\mathrm{~J}$ & $\mathrm{~N}-1$ & 0 \\
12 & $\mathrm{D}$ & $\mathrm{N}-1$ & 0 & 31 & $\mathrm{~K}$ & $\mathrm{~N}-2$ & 300 \\
13 & $\mathrm{D}$ & $\mathrm{N}-1$ & 0 & 32 & $\mathrm{~J}$ & $\mathrm{~N}-2$ & 800 \\
14 & $\mathrm{E}$ & $\mathrm{N}-2$ & 150 & 33 & $\mathrm{~V}$ & $\mathrm{~N}-2$ & 600 \\
15 & $\mathrm{~N}$ & $\mathrm{~N}-2$ & 300 & 34 & $\mathrm{~L}$ & $\mathrm{~N}-1$ & 0 \\
16 & $\mathrm{~T}$ & $\mathrm{~N}-2$ & 850 & 35 & $\mathrm{~L}$ & $\mathrm{~N}-1$ & 0 \\
17 & $\mathrm{D}$ & $\mathrm{N}-2$ & 750 & 36 & $\mathrm{Q}$ & $\mathrm{N}-2$ & 0 \\
18 & $\mathrm{~F}$ & $\mathrm{~N}-1$ & 0 & 37 & $\mathrm{M}$ & $\mathrm{N}-2$ & 500 \\
19 & $\mathrm{G}$ & $\mathrm{N}-2$ & 0 & 38 & $\mathrm{Q}$ & $\mathrm{N}-2$ & 0 \\
\hline
\end{tabular}

The training set includes 16 stable scenarios in order to train the protection not to act in stable cases. In the 22 unstable scenarios, the minimal load shedding $P_{i}^{*}$ has been determined as outlined in Section IV-A. The values, computed with an accuracy of $50 \mathrm{MW}$, are given in Table II. In this study, load shedding is shared out between 10 locations, each load being decreased proportionally to its initial value. As can be seen from Table II, the $P_{i}^{*}$ values range rather uniformly in the $\left[\begin{array}{ll}100 & 1450\end{array}\right] \mathrm{MW}$ interval, between marginally and severely unstable cases.

Requirements 1 to 4 of Section IV-B have been taken into account. In accordance with Hydro-Québec planning rules, the 3 rd requirement has been amended by allowing some (hopefully small) load shedding to take place after a stable but severe incident. The $\mathrm{N}-2$ scenario No 19 is concerned. The latter is handled as an unstable scenario with $P_{i}^{\star}=0$ in (8) and (9). This leads to finally processing $s=22+1=23$ scenarios. As regards the 4 th requirement, the average voltage $\bar{V}$ is not allowed to stay below $V_{1}^{\min }=0.94$ pu for more than 15 seconds.

\section{B. Search spaces}

Tables III and IV detail the two search spaces considered in this study, referred to as SP1 and SP2, respectively. The main difference between them concerns the number of unknowns to determine. In SP1, the parameters of rules $R_{1}, R_{2}$ and $R_{3}$ are fixed to values suggested by preliminary trial-and-error simulations. Hence, the parameters to be optimized (indicated with bold font) are those associated with the $R_{I}$ rule.

On the other hand, the purpose of SP2 is to let the methodology find the best parameters of rules $R_{1}, R_{2}$ and $R_{3}$ as well. The exponential complexity of the combinatorial optimization makes it nonetheless necessary to limit the number 
TABLE II]

SEARCH SPACE 1

\begin{tabular}{|c|c|c|c|c|}
\hline Rule & Param. & $\begin{array}{l}\text { Lower } \\
\text { bound }\end{array}$ & $\begin{array}{l}\text { Upper } \\
\text { bound }\end{array}$ & $\begin{array}{l}\mathrm{Nb} \text { of } \\
\text { values }\end{array}$ \\
\hline$R_{1}$ & $\begin{array}{c}V_{1}^{m i n}(\mathrm{pu}) \\
d_{1}(\mathrm{~s}) \\
\Delta P_{1}(\mathrm{MW})\end{array}$ & \multicolumn{3}{|c|}{$\begin{array}{c}0.94 \\
11 \\
400 \\
\end{array}$} \\
\hline$R_{2}$ & $\begin{array}{c}V_{2}^{\min }(\mathrm{pu}) \\
d_{2}(\mathrm{~s}) \\
\Delta P_{2}(\mathrm{MW})\end{array}$ & \multicolumn{3}{|c|}{$\begin{array}{c}0.93 \\
9 \\
400\end{array}$} \\
\hline$R_{3}$ & $\begin{array}{c}V_{3}^{m i n}(\mathrm{pu}) \\
d_{3}(\mathrm{~s}) \\
\Delta P_{3}(\mathrm{MW})\end{array}$ & \multicolumn{3}{|c|}{$\begin{array}{c}0.91 \\
6 \\
700\end{array}$} \\
\hline $\overrightarrow{R_{I}}$ & $\begin{array}{c}V_{\mathrm{I}}^{\min }(\mathrm{pu}) \\
\mathrm{d}_{\mathrm{I}}(\mathrm{s}) \\
\mathbf{k}_{(\mathrm{MW} / \mathrm{pu})} \\
\Delta \mathbf{P}_{\min }(\mathrm{MW}) \\
\Delta \mathrm{P}_{\max }(\mathrm{MW})\end{array}$ & $\begin{array}{c}0.94 \\
3 \\
5000 \\
0 \\
100\end{array}$ & $\begin{array}{c}0.95 \\
8 \\
15000 \\
400 \\
1000\end{array}$ & $\begin{array}{l}2 \\
6 \\
11 \\
5 \\
10\end{array}$ \\
\hline & $\begin{array}{l}\text { al number of } \\
\text { tances of } \mathbf{x}\end{array}$ & \multicolumn{3}{|c|}{5280} \\
\hline
\end{tabular}

TABLE IV

SEARCH SPACE 2

\begin{tabular}{|c|c|c|c|c|}
\hline Rule & Param. & $\begin{array}{l}\text { Lower } \\
\text { bound }\end{array}$ & $\begin{array}{l}\text { Upper } \\
\text { bound }\end{array}$ & $\begin{array}{l}\text { Nb. of } \\
\text { values }\end{array}$ \\
\hline \multirow[t]{3}{*}{$R_{1}$} & $\bar{V}_{1}^{\min }(\mathrm{pu})$ & \multicolumn{3}{|c|}{0.94} \\
\hline & $\mathrm{d}_{1}(\mathrm{~s})$ & 10 & 14 & 5 \\
\hline & $\Delta P_{1}(M W)$ & 100 & 400 & 4 \\
\hline \multirow[t]{3}{*}{$R_{2}$} & $V_{2}^{m i n}(\mathrm{pu})$ & \multicolumn{3}{|c|}{0.92} \\
\hline & $d_{2}(s)$ & \multicolumn{3}{|c|}{$d_{1}-4$} \\
\hline & $\Delta \mathrm{P}_{2}(\mathrm{MW})$ & 200 & 500 & 4 \\
\hline \multirow[t]{3}{*}{$R_{3}$} & $\bar{V}_{3}^{m i n}(\mathrm{pu})$ & \multicolumn{3}{|c|}{0.9} \\
\hline & $d_{3}(\mathrm{~s})$ & \multicolumn{3}{|c|}{$d_{1}-8$} \\
\hline & $\Delta P_{3}(M W)$ & 600 & 900 & 4 \\
\hline \multirow[t]{5}{*}{$\overline{R_{I}}$} & $\overline{V_{I}^{m z} n}(\mathrm{pu})$ & \multicolumn{3}{|c|}{0.95} \\
\hline & $d_{1}(s)$ & 3 & 8 & 6 \\
\hline & k (MW/pu) & 7000 & 15000 & 9 \\
\hline & $\Delta P_{\min }(\mathrm{MW})$ & 0 & 300 & 4 \\
\hline & $\Delta P_{\max }(\mathrm{MW})$ & 400 & 600 & 3 \\
\hline \multicolumn{2}{|c|}{$\begin{array}{l}\text { Total number of } \\
\text { instances of } x\end{array}$} & \multicolumn{3}{|c|}{77760} \\
\hline
\end{tabular}

of unknowns as well as the values tested for each of them. In this respect, the thresholds $V_{2}^{\text {min }}$ and $V_{3}^{\text {min }}$ have been chosen from the observation of the very first voltage drops (after the disturbance occurrence). They have also been chosen to that each rule is optimized from a significant number of scenarios. Finally, the size of the search space has been further reduced by "linking" the delays $d_{1}, d_{2}$ and $d_{3}$ as indicated in Table IV.

The total number of instances of $\mathbf{x}$ is given in both tables. The only considered designs are those satisfying inequalities (2), (3) and (4). Taking into account that each combination has to be tested over 23 scenarios, these figures confirm the huge complexity of the combinatorial optimization.

\section{Computing tools}

As of writing this paper, tests with detailed simulation are under progress. Therefore, we present hereafter the results of a preliminary study using QSS simulation.

To speed up computations, the dynamic reordering technique mentioned in Section IV-C has been used.

Finally, when optimizing the $L_{1}$ objective from SP2, the computations have been distributed over 4 PCs, according to the second scheme of section IV-D

\section{Results and discussion}

Table V shows, for each search space and each objective, the optimal value found for the various parameters and the value of the corresponding objective. For information only, the value of the other (non optimized) objective is given in parentheses underneath the first one. Let us emphasize that all four requirements of Section IV-B are met in all designs of Table V.

TABLE $V$

OPTIMIZATION RFSULTS

\begin{tabular}{|c|c|c|c|c|}
\hline & \multicolumn{2}{|c|}{ SPI } & \multicolumn{2}{c|}{ SP2 } \\
& $L_{\infty}$ & $L_{1}$ & $L_{\infty}$ & $L_{1}$ \\
\hline$V_{1}^{\text {min }}(\mathrm{pu})$ & 0.94 & 0.94 & 0.94 & 0.94 \\
$d_{1}(\mathrm{~s})$ & 11 & 11 & 10 & 10 \\
$\Delta P_{1}(\mathrm{MW})$ & 400 & 400 & 100 & 100 \\
\hline$V_{2}^{\text {min }}(\mathrm{pu})$ & 0.93 & 0.93 & 0.92 & 0.92 \\
$d_{2}(\mathrm{~s})$ & 9 & 9 & 6 & 6 \\
$\Delta P_{2}(\mathrm{MW})$ & 400 & 400 & 200 & 200 \\
\hline$V_{3}^{\min }(\mathrm{pu})$ & 0.91 & 0.91 & 0.9 & 0.9 \\
$d_{3}(\mathrm{~s})$ & 6 & 6 & 2 & 2 \\
$\Delta P_{3}(\mathrm{MW})$ & 700 & 700 & 600 & 600 \\
\hline$V_{I}^{\min }(\mathrm{pu})$ & 0.94 & 0.95 & 0.95 & 0.95 \\
$d_{I}(\mathrm{~s})$ & 3 & 3 & 3 & 3 \\
$k$ MW/pu) & 15000 & 11000 & 7000 & 7000 \\
$\Delta P_{\min }(\mathrm{MW})$ & 300 & 100 & 300 & 200 \\
$\Delta P_{\max }(\mathrm{MW})$ & 400 & 400 & 400 & 400 \\
\hline Objective & 400 & 167 & 400 & 148 \\
value & $(196)$ & $(600)$ & $(170)$ & $(600)$ \\
\hline
\end{tabular}

As can be seen, the performances of the controllers found from the two search spaces are the same when the $L_{\infty}$ objective is considered (see 2 nd and 4 th columns of the table). On the other hand, optimizing the $L_{1}$ objective from SP2 allows to find a better design (see 3 rd and 5th columns). This result indicates that, while the primary objective of rules $R_{1}$ to $R_{3}$ is to make voltages promptly recover, they are also useful to improve the controller performances in terms of shed amounts. The final choice between the two objectives will be made from the results of detailed simulations.

Figures 6 and 7 show two typical time evolutions of $\bar{V}$ for onc of the obtained designs.

In Fig. 6, the behaviour is typical of a "traditional" undervoltage load shedding scheme. In the first seconds following the occurrence of the disturbance, several rules are activated to shed an important amount of load, in order $\bar{V}$ to be brought back above $0.94 \mathrm{pu}$. The final stabilization is then guaranteed by MAIS devices and the $R_{I}$ rule.

The situation is different in Fig. 7: although $\bar{V}$ falls below $V_{1}^{\text {min }}$ and $V_{2}^{\text {min }}$ just after the disturbance, the controller takes advantage of the MAIS devices, which make $\bar{V}$ recover above $0.94 \mathrm{pu}$. Once the voltage decrease resumes, the controller has to wait for rule $R_{1}$ to shed $400 \mathrm{MW}$; then, it relies on rule $R_{I}$ to adjust its action to the severity of the situation. This allows the total load shed $(700 \mathrm{MW})$ to be very close to the target value $\left(P_{i}^{\star}=650 \mathrm{MW}\right)$. 


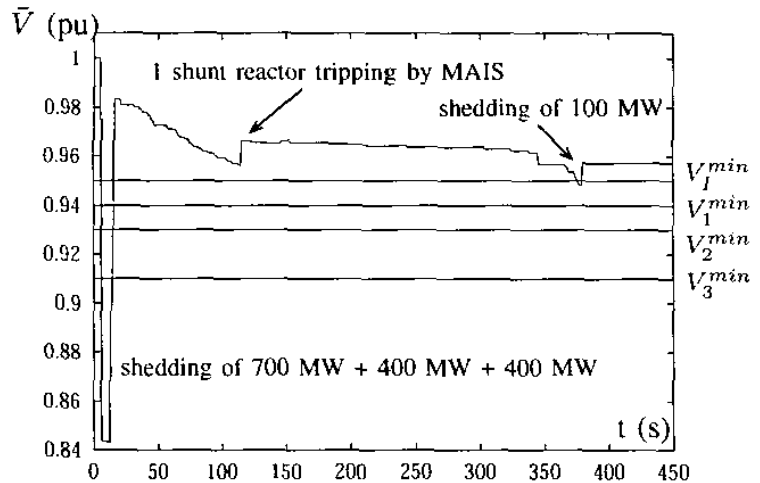

Fig. 6. Time evolution of $\bar{V}$ in scenario 26

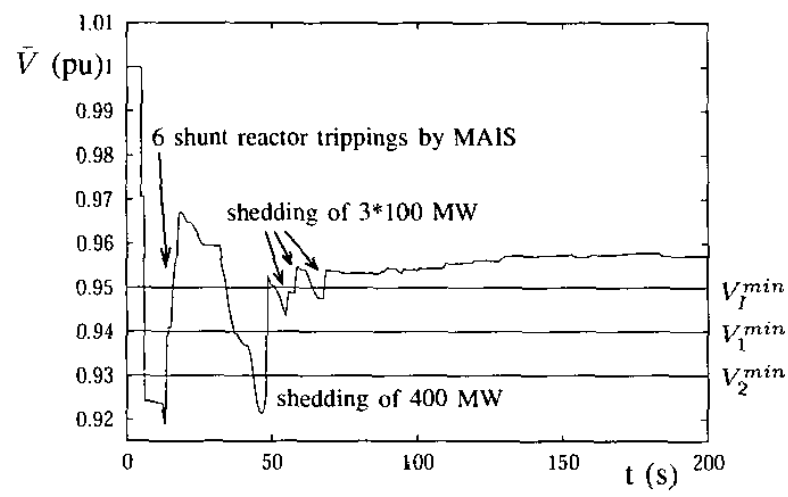

Fig. 7. Time evolution of $\bar{V}$ in scenario 28

Table VI compares the total number of simulations required to design the controller from the two search spaces. These figures confirm that optimizing the $L_{1}$ objective requires more simulations and hence is more time consuming. The gain with respect to a brute force enumeration is much more important in the case of $L_{\infty}$, as discussed in [6].

However, the optimization of $L_{1}$ from SP2 shows an interesting result: sharing out the search space between several processors (which was not done for SP1) allows to break more enumerations and hence reject a larger percentage of (useless) designs. Indeed, with 4 processors working in parallel, lower values of $I_{b}$ have been discovered sooner, and broadcasted to the other processors. From the computing time viewpoint, this adds to the gain naturally obtained from parallel computations. These two speed-ups cumulated bring an overall gain in computing time of 15 .

\section{CONCLUSION}

This paper has described some characteristics of TDST, an undervoltage load shedding scheme scheduled for implementation in the Hydro-Québec system by the beginning of 2004 . The emphasis was on the control logic. Obviously, many other practical aspects, essential for the reliability of such a system
TABLE VI

TOTAL NUMBER OF SIMULATIONS REOUIRED FOR THF OPTIMIZATIONS

\begin{tabular}{|c|c|c|c|c|}
\hline & $L_{\infty}$ SP1 & \multicolumn{2}{|c|}{ SP2 } \\
\hline $\begin{array}{c}\text { Brute force } \\
\text { enumeration }\end{array}$ & 121440 & 121440 & 1788480 & 1788480 \\
\hline $\begin{array}{c}\text { B\&B } \\
\text { approach }\end{array}$ & 7773 & 101322 & 98242 & 331479 \\
\hline Gain & 16 & 1.2 & 18 & 5.4 \\
\hline
\end{tabular}

protection scheme, have not been considered here.

TDST will rely on four if-then rules. Three of them aim at making the average voltage promptly recover above some admissible level, and will make up the first line of defence against voltage instability. Conditioned to the previous triggering of these rules, the fourth one is allowed to act several times and to adjust its action to the severity of the situation, thereby yielding a robust closed-loop protection.

The combinatorial optimization (Branch-and-Bound) methodology previously proposed by the authors to optimize the parameters of the above rules, has been applied to this new structure, using a new set of dimensioning scenarios. The results of a preliminary study have been presented in this paper. Tests with detailed simulations are under progress. Distributed processing, as outlined in this paper, is essential to keep the optimization approach tractable while investigating a sufficiently large search space for the protection parameters.

Obviously, the final tuning of TDST will have to take into account several other aspects, such as the coordination with the existing RPTC.

The method is already useful to assist Hydro-Québec engineers in the highly combinatorial and multi-faceted task of adjusting TDST parameters. It will also prove useful for updating the protection parameters, for instance when transmission system reinforcements will come into effect.

\section{REFERENCES}

[1] C. W. Taylor, Power System Voltage Stability, McGraw Hill, EPRI Power System Engineering series, 1994

[2] T. Van Cutsem, C. Vournas, Voltage Stability of Electric Power Systems, Boston, Kluwer Academic Publishers, 1998

[3] C. W. Taylor, "Concepts of undervoltage load shedding for voltage stability", IEEE Trans, on Power Delivery, Vol. 7, pp. 480-488, 1992

[4] C. Moors, D. Lefebvre, T. Van Cutsem, "Load Shedding Controllers Against Voltage Instability: a Comparison of Designs", Proc. of the IEEE Power Tech Conference, Porto (Portugal), 2001, ISBN 0-7803-7140-2

[5] C. Moors, D. Lefebvre, T. Van Cutsem. "Design of load shedding schemes against voltage instability", IEEE Power Engineering Society Winter Meeting, Singapore, 2000

[6] C. Moors, On the Design of Load Shedding Schemes against Voltage Instability in Electric Power Systems, PhD thesis, University of Liège, Fac. of Applied Sciences, October 2002

[7] S. Bemard, G. Trudel, G. Scott, "A 735-kV shunt reactors automatic switching system for Hydro-Québec network", IEEE Trans. on Power Systems, Vol. 11, pp. 2024-2030, 1996

[8] CIGRE Working Group 34-02-19, System Protection Schemes in Power Networks, CIGRE publication, 2001

[9] W. S. Cook, W. H. Cunningham, W. R. Pulley Blank, A. Schrijver, Combinatorial Optimization, Wiley-Interscience, 1998

[10] L. Loud, P. Rousseaux, D. Lefebure, T. Van Cutsem, "A time-scale decomposition-based simulation tool for voltage stability analysis", Proc. of the IEEE Power Tech Conference, Porto (Portugal), 2001, ISBN 07803-7140-2. 